Africa of archives for the preservation of these recordings-this was given the highest priority by the committee; (3) to accept a preliminary outline of the periodization and regionalization of African history; (4) to invite the co-operation of other disciplines; (5) to promote the publication of teaching aids as well as materials of specialist interest; $(6)$ to further co-operation between UNESCO and institutes of African studies; (7) to create a permanent consultative committee; (8) to begin a summary of our knowledge of the nineteenth century, which was recognized as the most researched period at the moment, and to encourage research in a number of more neglected areas.

The recommendations were submitted to the fourteenth session of the General Conference of UNESCO, which met in October 1966.

\title{
Un Colloque international d'Archéologie africaine à Fort-Lamy
}

De nombreux archéologues venus d'Afrique du Nord, d'Afrique occidentale, d'Afrique centrale, d'Afrique orientale, de France, d'Allemagne, d'Italie et des États-Unis, principalement, se sont réunis à l'Institut national tchadien pour les Sciences humaines de FortLamy du i i au i6 décembre 1966.

Les participants à ce colloque, organisé par l'UNESCO et le Gouvernement de la République du Tchad avec le patronage scientifique du Centre national de la Recherche scientifique (Paris), étaient les suivants : Mlle Aumassip (Centre de Recherches anthropologiques, préhistoriques et ethnographiques, Alger), M. Awad Saadawia (Direction des services libyens d'archéologie), M. G. Bailloud (CNRS), M. le Doyen L. Balout (Université de Paris et Muséum national d'Histoire naturelle), M. G. de Beauchêne (IFAN, Niger), M. B. Blankoff (Société de préhistoire du Gabon), M. le Professeur et Mme Camps (Université et Centre de Recherches anthropologiques, préhistoriques et ethnographiques d'Alger), M. Brahim Benoît (INTSH), M. J. Chapelle (INTSH et Musée national tchadien), M. G. E. Connah (Université d'Ibadan), M. le Professeur C. S. Coon (Université de Philadelphie), Dr. Y. Coppens (CNRS), M. Courtin (CNRS), M. Ekpo Eyo (Directeur Adjoint des Antiquités, Nigeria), M. Gamacchio (Fondation Lerici, Milan), M. J. Gauthier (CNRS), Dr. J. H. Hugot (IFAN, Dakar), M. Hurel (UNESCO), M. Jauffret (Directeur des Services géologiques, Fort-Lamy), M. le Professeur J. Leclant (Université de Paris), M. le Professeur et Mme Lebeuf (CRNS), M. H. Lhote (CNRS et Musée de l'Homme), M. le Professeur Monod, Membre de l'Académie des sciences (Muséum national d'Histoire naturelle), Dr. R. B. Nunoo (Directeur du Musée national ghanéen), Dr. Posnansky (Directeur des études africaines, Kampala), M. J. P. Roset (ORSTOM), Mme Roubet (Centre de Recherches anthropologiques, préhistoriques et ethnographiques, Alger), M. Rollando (France), Dr. Schläger (Institut allemand d'Archéologie, Rome), M. Schneider (B.R.G.M., FortLamy), M. le Professeur Thurstan Shaw (Université d'Ibadan), M. le Dr. Ziegert (Institut allemand de Bardaï).

Ce colloque, qui constitue une entreprise de groupement régional du même ordre que le récent Séminaire d'archéologie de Freetown (28 et 29 juin 1966) représenté à Fort-Lamy par le Professeur Thurstan Shaw, a fait état des recherches poursuivies dans une grande partie de l'Afrique. Il a rédigé des vœux à soumettre aux gouvernements intéressés et des recommandations destinées aux organismes de recherches spécialisés en insistant tout particulièrement sur l'importance de l'enseignement de la préhistoire africaine et de son intégration à la formation générale des historiens de l'Afrique, sur la réunion régulière de colloques régionaux et sur l'urgence de la formation intensive d'archéologues nationaux africains. Il a travaillé en liaison avec les Congrès internationaux de préhistoire et d'étude du quaternaire qui étaient représentés au Colloque de Fort-Lamy par M. le Doyen L. Balout. 